\title{
Heartbeat: Social isolation is associated with increased mortality after acute myocardial infarction or stroke
}

doi:10.1136/heartjnl-2018-313989

Loneliness and social isolation are associated with an increased risk of premature mortality, yet the specific diseases contributing to these adverse outcomes have not been fully elucidated. In 2016, Heart published a meta-analysis that showed 'Poor social relationships were associated with a $29 \%$ increase in risk of incident coronary heart disease (pooled relative risk: $1.29,95 \%$ CI 1.04 to 1.59 ) and a $32 \%$ increase in risk of stroke (pooled relative risk: $1.32,95 \%$ CI 1.04 to 1.68$).{ }^{1}$ This paper and the linked editorial ${ }^{2}$ received some of the highest Altmetric scores for all papers published in Heart, reflecting the importance of this topic to the general public and healthcare systems. However, while a meta-analysis is a powerful approach to combining data, published studies do not always provide enough detail about other factors that might affect these findings and thus is difficult to translate into patient care or public policy.

In this issue, the association of loneliness and social isolation with cardiovascular disease (CVD) risk is examined in further detail in a UK Biobank cohort of over 479000 men and women. ${ }^{3}$ Although unadjusted data suggested that social isolation and loneliness were associated with a significant higher risk of acute myocardial infarction (AMI) and stroke, the detailed information available in the data set allowed adjustment for other known cardiovascular risk factors. In fact, after correction for other risk factors, these associations were significantly attenuated and were no longer statistically significant for the overall cohort. The relative importance of adjustment for health behaviours, depressive symptoms, socioeconomic and other risk factors is shown in figure 1 . However, in the subgroup of patients who suffered an AMI or stroke, the fully adjusted model did show that social isolation, but not loneliness, was associated with increased mortality with HR 1.25 (95\% CI 1.03 to 1.51) for those with an AMI and 1.32

Correspondence to Professor Catherine M Otto, Division of Cardiology, University of Washington, Seattle,WA 98195, USA; cmotto@uw.edu

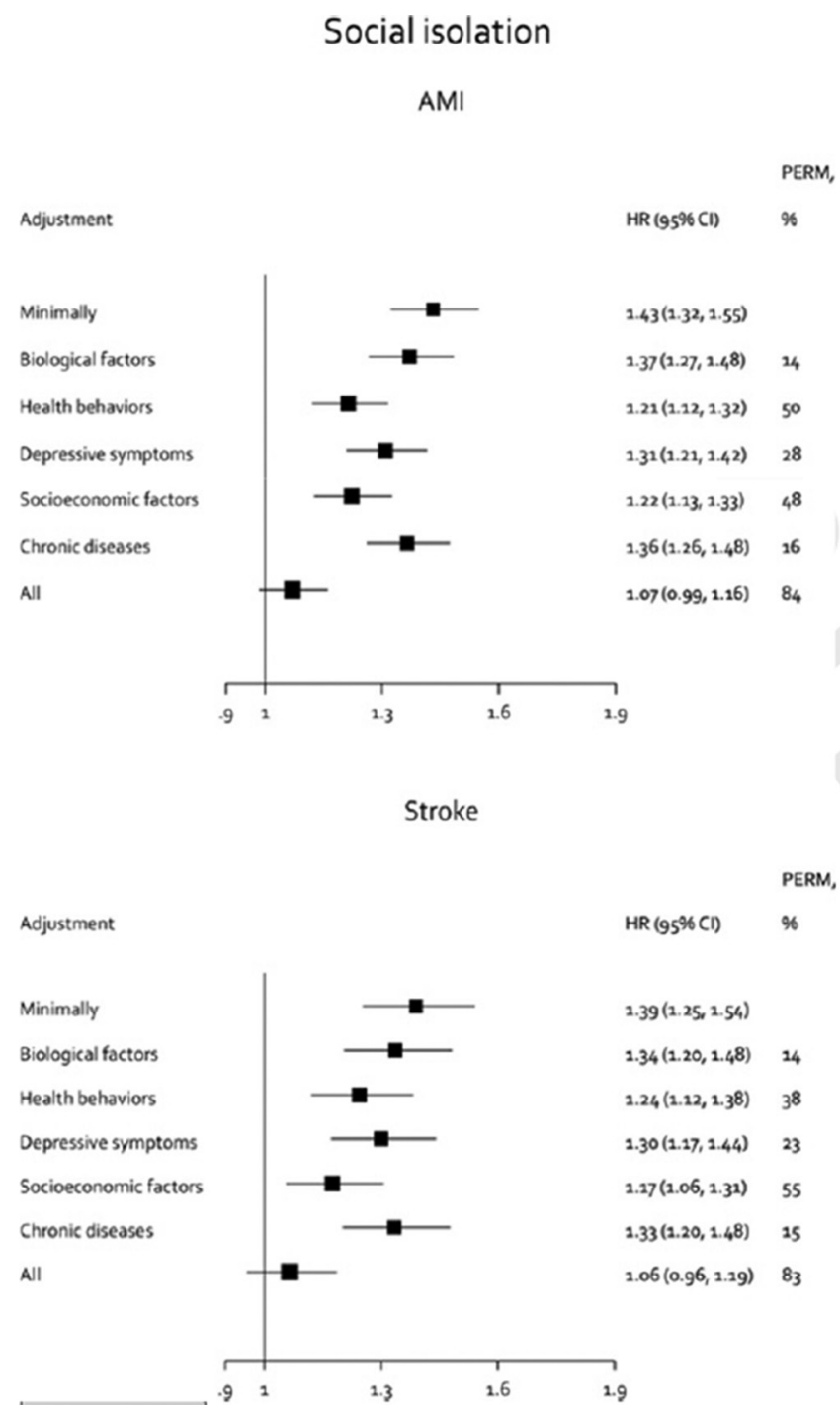

Figure 1 Entire Biobank cohort $(n=479$ 054). Proportions of the social isolation-AMI and stroke excess risk mediated by biological, behavioural, socioeconomic and health-related factors. AMI, acute myocardial infarction; PERM, percentage of excess risk mediated.

(95\% CI 1.08 to 1.61 ) for those with a stroke (figure 2).

This important study demonstrates the additive CVD risk associated with social isolation after AMI or stroke and thus identifies a group of patients where efforts to reduce social isolation would be likely to have the most effect. The attenuation of risk with adjustment for other known risk factors also emphasises the key role of these linked factors, particularly patient health behaviours, in reducing CVD risk.

Detection of asymptomatic atrial fibrillation (AF) is an increasing public health concern given the increased prevalence of AF in our ageing population and the stroke risk associated with this cardiac rhythm. Various screening approaches have been proposed in papers previously published in Heart, including use of smartphone ECGs 


\section{Social isolation}

Mortality after AMI

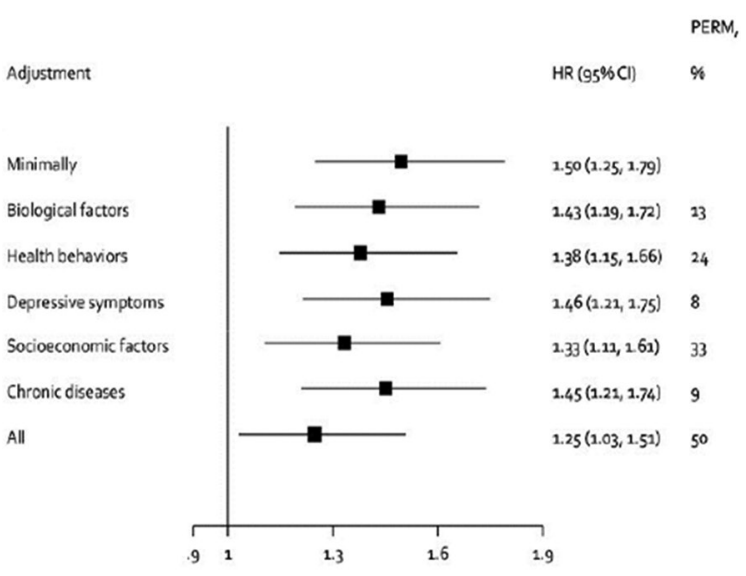

Mortality after stroke

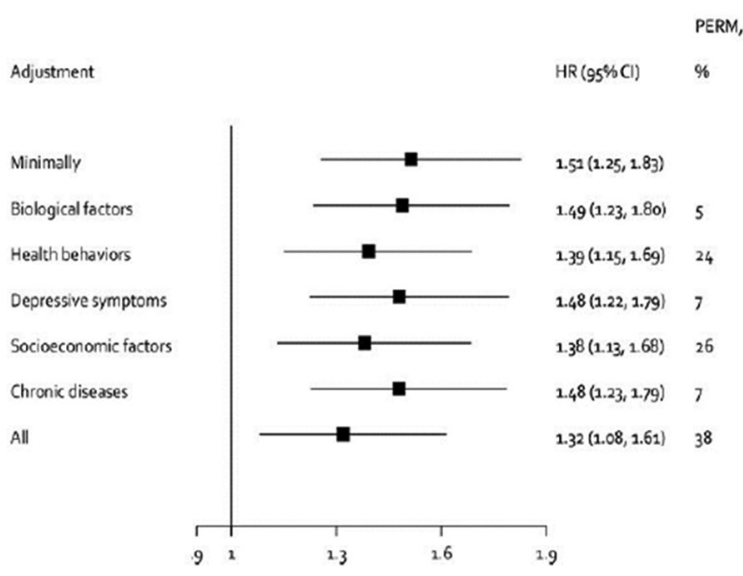

Figure 2 Partcipants with incident AMI or stroke $(n=9202)$. Proportions of the social isolationmortality after AMI or stroke event excess risk mediated by biological, behavioural, socioeconomic and health-related factors. AMI, acute myocardial infarction; PERM, percentage of excess risk mediated.

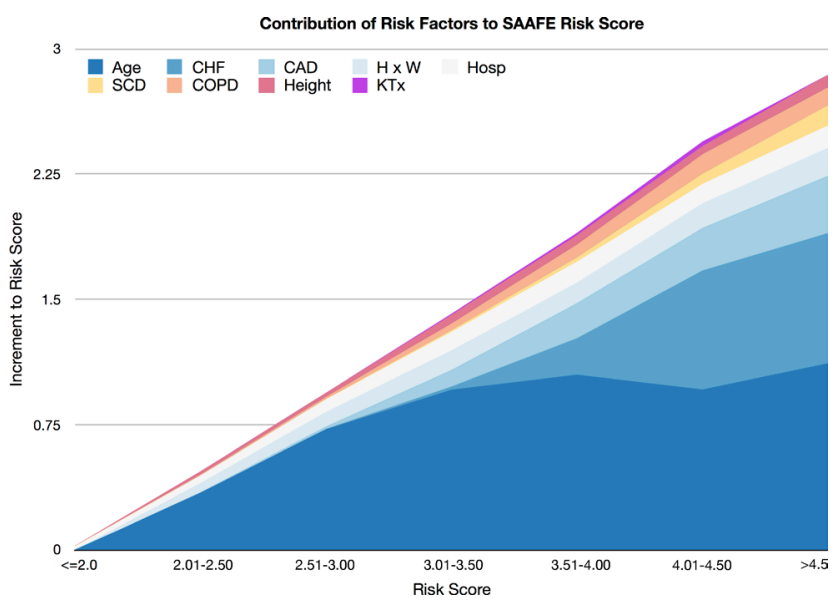

Figure 3 Contribution of risk factors to total Screening for Asymptomatic Atrial Fibrillation Events (SAAFE) risk score. The variables are age, $C H F, C A D, H \times W$, recent hospitalisation for cardiopulmonary conditions (Hosp), cardiac arrest (SCD), chronic obstructive lung disease (COPD), height and KTx. Note that the first three variables have the greatest contribution to the total risk score. $C A D$, coronary artery disease; $C H F$, congestive heart failure; $C O P D$, chronic obstructive pulmonary disease; $\mathrm{H} \times \mathrm{W}$, height times weight; $\mathrm{KTx}$, kidney transplant; $\mathrm{SCD}$, sudden cardiac death. in the general population ${ }^{4}$ and handheld ECG devices in hospitalised geriatric patients, ${ }^{5}$ to name just a few. ${ }^{6}$ Linker and colleagues ${ }^{7}$ propose a new risk score based on clinical variables, without an ECG, to identify patients at risk of AF development. The clinical risk factors included in the Screening for Asymptomatic Atrial Fibrillation Events risk score and the contribution of each to the overall model are shown in figure 3. The risk of AF increased from a low of $2 \%$ in those with a score of 2.0 or less to a high of $66 \%$ in those with a score $>4.5$. Overall, the model identified $81 \%$ of patients with incident AF over 3 years.

In an editorial, Bisson and colleagues ${ }^{8}$ agree that lower cost screening methods for AF are needed but also suggest, 'Another strategy might be to propose early oral anticoagulation in patients with very high risk of AF and of embolic ischaemic stroke, before AF itself is documented. Further knowledge on implication of multiple risk factors in patients without AF is thus warranted and might influence future strategies in stroke prevention (figure 4). The major challenge will be to find a cost-effective prophylaxis with a favourable risk-benefit ratio, which will require randomised trials in such high-risk patients.'

The Education in Heart article $^{9}$ summarises the investigation and management of adults with hypertension. Hypertension treatment might seem mundane but effective blood pressure control is essential for prevention of CVD and stroke. Identification of patients with secondary hypertension allows intervention to correct the underlying cause but diagnostic testing is indicated only when clinical characteristics suggest this diagnosis. Recommendations for initiation of therapy and long-term management have been developed by several professional organisation and are summarised in a concise readable format in this article (figure 5).

The Image Challenge asks you to interpret the ECG in a patient with a recently implanted pacer. ${ }^{12}$ The ECG seems straightforward at first glance but there is more than meets the eye.

Competing interests None declared.

Provenance and peer review Commissioned; internally peer reviewed.

(C) Author(s) (or their employer(s)) 2018. No commercial re-use. See rights and permissions. Published by BMJ. 


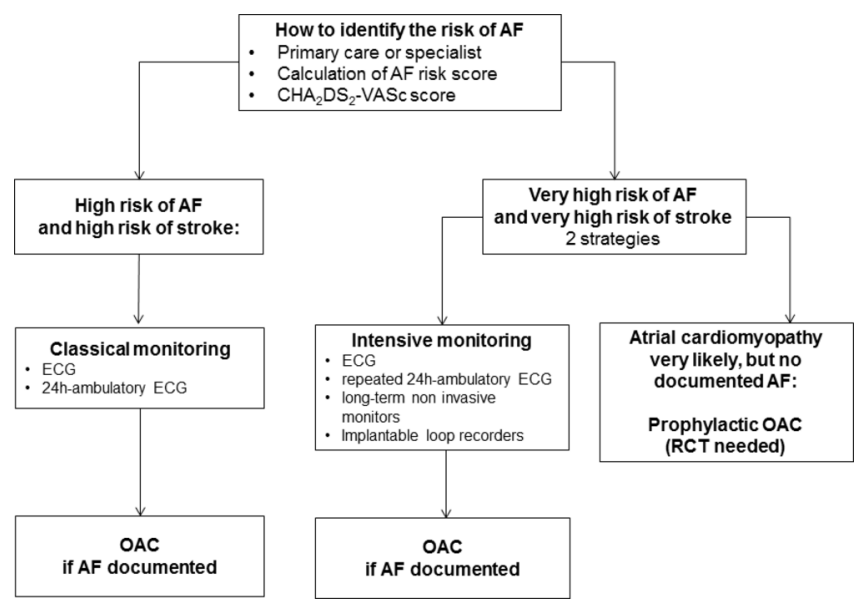

Figure 4 Possible strategies to be proposed or evaluated in the future when using screening tools identifying patients at risk for $\mathrm{AF}$ in clinical practice. $\mathrm{AF}$, atrial fibrillation; $\mathrm{CHA}_{2} \mathrm{DS}_{2}-\mathrm{VASC}$, congestive heart failure, hypertension, age ( $\geq 75$ years; 2 points), diabetes, stroke/transient ischaemic attack ( 2 points), vascular disease, age (65-74 years), sex category (female); OAC, oral anticoagulation; RCT, randomised controlled trial.

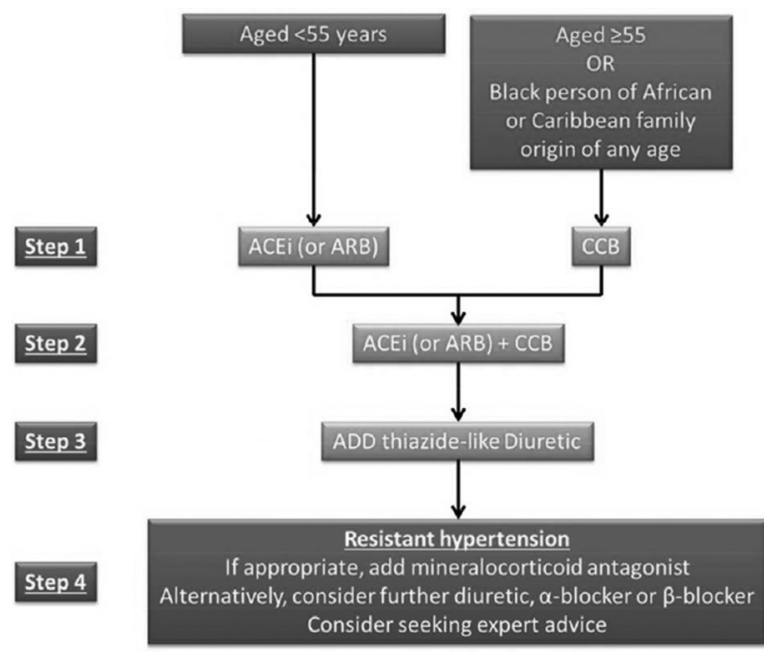

Figure 5 Flow diagram for selection of class of antihypertensive pharmacotherapy (Adapted from BHS/NICE guidelines [10] and incorporating additional evidence from Williams et al [11]). However, see online supplementary table 2 for the rationale against an all-purpose ranking of drugs. ACEi, ACE inhibitor; ARB, angiotensin receptor blocker; BHS, British Hypertension Society; CCB, calcium channel blocker; NICE, National Institute for Health and Care Excellence.
Check for updates

To cite Otto CM. Heart 2018;104:1471-1473.

Heart 2018:104:1471-1473

doi:10.1136/heartjnl-2018-313989

\section{REFERENCES}

1 Valtorta NK, Kanaan M, Gilbody S, et al. Loneliness and social isolation as risk factors for coronary heart disease and stroke: systematic review and metaanalysis of longitudinal observational studies. Heart 2016;102:1009-16.

2 Holt-Lunstad J, Smith TB. Loneliness and social isolation as risk factors for CVD: implications for evidence-based patient care and scientific inquiry. Heart 2016;102:987-9.

3 Hakulinen C, Pulkki-Råback L, Virtanen M, et al. Social isolation and loneliness as risk factors for myocardial infarction, stroke and mortality: UK Biobank cohort study of 479054 men and women. Heart 2018;104:1536-42.

4 Chan NY, Choy CC. Screening for atrial fibrillation in 13122 Hong Kong citizens with smartphone electrocardiogram. Heart 2017;103:24-31.

5 Tavernier R, Wolf M, Kataria V, et al. Screening for atrial fibrillation in hospitalised geriatric patients. Heart 2018:104:588-93.

6 Keach JW, Bradley SM, Turakhia MP, et al. Early detection of occult atrial fibrillation and stroke prevention. Heart 2015;101:1097-102.

7 Linker DT, Murphy TB, Mokdad AH. Selective screening for atrial fibrillation using multivariable risk models. Heart 2018;104:1492-9.

8 Bisson A, Bodin A, Fauchier L. Why and how to screen for atrial fibrillation. Heart 2018;104:1474-5.

$9 \mathrm{Ng} \mathrm{FL}$, Lobo MD. Investigation and management of adult hypertension. Heart 2018;104:1543-51.

10 National Institute for Health and Care Excellence. Hypertension in adults: diagnosis and management Clinical guideline: NICE, 2011.

11 Williams B, MacDonald TM, Morant S, et al. Spironolactone versus placebo, bisoprolol, and doxazosin to determine the optimal treatment for drug-resistant hypertension (PATHWAY-2): a randomised, double-blind, crossover trial. Lancet 2015;386:2059-68.

12 Chow CL, Abu Baker B, Mohamed U. Pacing: a new look. Don't be deceived. Heart 2018; 104:1491. 